\title{
Erratum: Compliance or Security, What Cost? (Poster)
}

\author{
Craig Wright \\ Springer-Verlag, Computer Science Editorial, Tiergartenstr. 17, \\ 69121 Heidelberg, Germany \\ \{cwrigh20\}@postoffice.csu.edu.au
}

U. Parampalli and P. Hawkes (Eds.): ACISP 2011, LNCS 6812, pp. 412-416, 2011.

(C) Springer-Verlag Berlin Heidelberg 2011

DOI 10.1007/978-3-642-22497-3_36

In the original version the author affiliation was wrongly added in this paper. It should read as: Charles Sturt University, Australia 